\title{
Design of Polar Codes for Rayleigh Fading Channel using Random Interleaver and De- Interleaver
}

\author{
T.Arunajyothi, S.V.R.K.Rao
}

\begin{abstract}
Rayleigh fading channel in polar codes based on random interleaver and de-interleaver is presented in this paper. Multipath fading channels are obtained by inducing the sub channels based on the transformation of polarizations. This will track the noise and diversity in the channels. The performance of polar code is improved by constructing the dynamic frozen symbol and the Nakagami channel is introduced in estimating diversity system. Random inter leaver and deinterleaver will permute the signals in effective way. Code construction will allow the system to map the address of memory location. Here the polar code encoding system will analyze the characteristics of channels. The results show that effective output is obtained based on polar code fading channel.
\end{abstract}

KEY WORDS: - Polar codes, fading channel, Nakagami Channel, Rayleigh fading channel.

\section{INTRODUCTION}

Now a day, communication plays a major role in transmitting the channels based on capacity and performance. To increase the capacity of antenna in cooperative diversity, various bandwidths are specified. The combination of signals will relay on the diversity. In the same way multi hop signals are used which will rely directly from networks [1]. Binary input memories less output symmetric channels are used in the polar code to encode and decode the system. This system gives effective output compared to others. Generally to construct the polynomial function, some algorithms are introduced which produce complexity in the system. Polarization transformation is introduced to detect the polar code channel and this transformation gives better performance in the form of bits.

Basically, sub channels uses bit error probability for detecting errors. Code designed will decode the algorithm to obtain low and average complexity performance. While transmitting the channels, multiple signal paths are introduced in real world wireless communications. Fading channels are obtained because of variation in time. Hence the polar code will transmit the channels in effective way.

Here, Rayleigh fading channels are introduced in the polar code to distribute the channel information. The channel information distributes based on both transmitter and receiver. Both polar code and polar lattice is independently constructed to fade the channels. Symmetric channels are introduced in the form of binary and this is done in polar coding construction [2]. The Rayleigh fading channel based polar code will assume the channel depend on

Revised Manuscript Received on August 14, 2019.

T.Arunajyothi, PG Scholar, Department of ECE, Godavarai institute of Engineering and Technology, Rajahmundry, Andhra Pradesh, India.

Dr.S.V.R.K.Rao, Professor, Department of ECE, Godavarai institute of Engineering and Technology, Rajahmundry, Andhra Pradesh, India. the statistics performance. Channel estimation is done depending on the limitations performed. Error correction performance is maintained in statistical format which is unreliable. At the receiver, the channel estimation is specifically determined and polar code is also designed using fading channels.

The main issue in the polar code is to solve the unsolved error probability occurred from sub channel. The upper and lower bounds are tracked based on the approach used. Here the bounds which are bounded will not be guaranteed. Code constructions are performed using two states. Fading AWGN channel is approximated depend on the symmetrical channel [3].

Sub channels in Rayleigh fading gives good performance based on the Monte-Carlo simulations. This will distribute the channel to get good performance. Bit error probability is evaluated in this system by the transformation of channel. Compared to turbo codes and LDPC codes, polar code will give effective results. Polar code will decode and encode the system based on the functionality. Decoding technique will be analyzed based on the sequential generalization Information symbols are introduced between the systems to solve the problem. Error-correcting or error-detecting code will enable the code based on the operation performed. In this paper, polar code is developed to moderate the system performance compared to nakagami channel. By transforming the fading channels, the polarization is performed. All the equations in the system will satisfy the requirements sequentially and numerically.

\section{RELATED WORK}

Here $\mathrm{N}$ independent sources are used in constructing the polar code and this is equal to ones set of operation. In the other set of channel the polarizing effect is sensed. The capacity of polar code is varnished depending on two logics they are 0 or 1 . Here channel splitting and channel combination is done based on the phase that is determined. Linear block codes will determine the bits of error correction in effective way. The generator matrix will determine the system performance. The generator matrix consists of code length $\mathrm{N}$ or $2 \mathrm{n}$. Hence by using generator matrix length of code is determined and in the same way, kernel matrix $\mathrm{F}$ is used to determine the power. Both input bits and frozen bits are used to transmit the information in the system. The generator matrix will ON the code word 


\section{DESIGN OF POLAR CODES FOR RAYLEIGH FADING CHANNEL USING RANDOM INTERLEAVER AND DE-INTERLEAVER}

block for the system operation. By using the equation $\mathrm{O}=\mathrm{I}$ * GN, generator matrix is determined. This equation will be in vector form. Here I is nothing but input information and $\mathrm{O}$ is nothing but output information. Channel polarization is performed based on the transformation that is done. Iterative application is done based on step polar transform. Good channels are obtained through polar codes for better communication. Here good channels are indicated with the value 1 and bad channels are indicated by 0 . By using this both encoder and decoder channels are determined.

Binary input memory less channels are used to obtain the capacity of polar coding. Noisy channels are estimated by using the code length based on collective channel. Here N channels are estimated depend on the system that is determined. The two input samples are used for the parallel processing applications. Adequate polar code is determined based on the cyclic redundancy check. Polar code is determined based on the length of the system and hardware density [4].

Block wise decoding process is performed busing linear block codes. This will perform the operations in a sequential manner. Some parameters are used to decode the system. Here polar code will determine the path search technique using SC, SCL and SCS decoding. By mixing all these parameters like SCS, SCL, new successive cancellation hybrid logic is obtained. In polar code, the encoder will use the partial sum products to access the system. SRAM is used to store the data path. The memory usage and area is maintained to reconstruct the multi level quantization.

A special construction is introduced by the Arikan, which will determine the theoretical problems. Here length is generated based on the problems that are detected. The capacity of the system will be utilized depend on the random coding method. The code size is increased based on the usage of memory and XOR gates. To increase the throughput of system, polar code decoder will be analyzed. The latency and hardware difficulty problems are solved by using polar code encoder and decoder.

In CMOS technology, the design is synthesized using parallel structure. Low latency and high throughput is obtained using the parallel structures. Polar cosine transform algorithm is introduced to divide the patches and extract the features. Hence image encryption and decryption unit is performed based on the polar coding. Here the information is mapped in the form of butterfly unit by using folding transformation. Repetitive operations are performed in digital signal processors. Single frame and subsequent frame is introduced to specify the life time of the system [5].

\section{EXISTED SYSTEM}

From below figure (1), the operation of existed system is observed. Here in first stage, the data is encoded using input parameter d. same encoding circuit is used for the first and second half of the circuit. The process of polarization performed is fast. Here sub channels are estimated based on the properties that are achieved. The selected sub channels is represented by using $\mathrm{W}(\mathrm{i}) \mathrm{N}$ \}. This system consists of mutual information. The sub channels are selected based on the criterion that is chosen. Sub channels are selected to get low complexity results. Here the ith bit is eliminated based on the node determination done. From figure (1), the information of bits are represented in the tree format.

Basically, a vector $y$ is received to rearrange the elements and this gives output $y$ as o. this condition is nothing but to start the process and reduce the $\mathrm{q}$ array symbols. By using joint density functions, the q-array is determined. From left end of the graph we can observe that size of two bits are equal for decoding process. The two transformation parameters will be used that is $\mathrm{d}$ and $\mathrm{d} 0$.according to the given condition, the operation is performed.

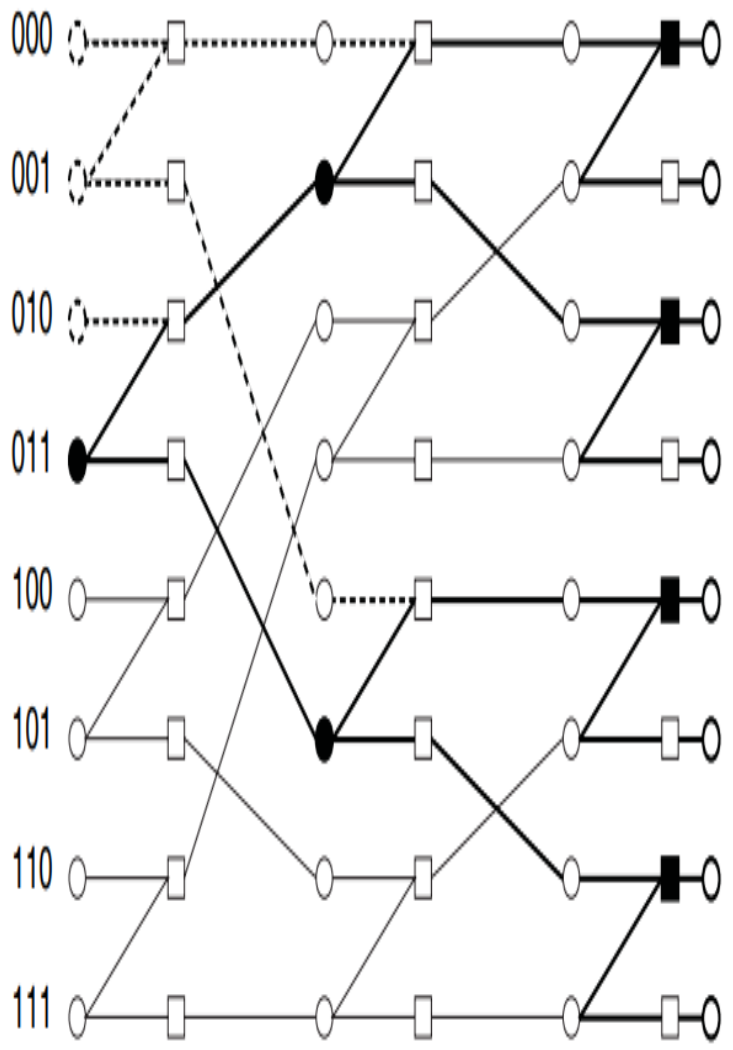

Fig. 1: EXISTED SYSTEM

By using density transformation, the equations of transformation will be determined. Here the complexity of the system will increases but it will reduce the size factor and latency of the system. To reduce the dimension of graph, multiple folding techniques are used. The dimensions will be in the range of $\mathrm{N} / 2 \mathrm{k}$. parallel processing is performed to reduce the latency of factor by $2 \mathrm{k}$ bits.

The q-array interpretation is performed in the vector form using vectors. Q-array will decode the symbol based on parallel operation. The both encoding and decoding process is introduced in the proposed system to get effective output. From below figure (2) we can observe the graph of signal to noise ratio of the system. 


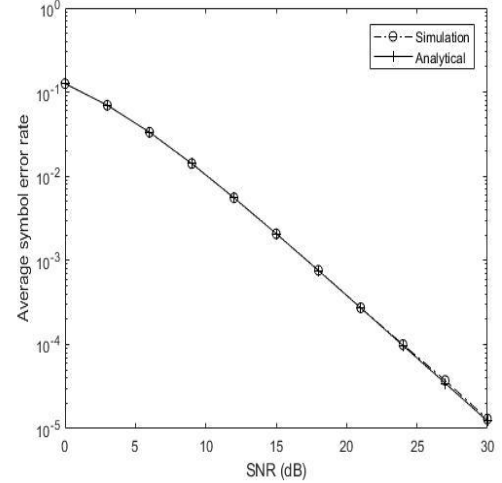

Fig. 2: COMPARISION OF ANALYTICAL \& SIMULATED OUTPUT OF ERROR RATE AND SNR OF NAKAGAMI FADING

\section{PROPOSED SYSTEM \& RESULTS}

The below figure (3) shows the block diagram of proposed system. In this system polar encoder, polar decoder, random interleaver, one dimensional and two dimensional methods, code construction and channel is used. Here the channel will estimate the threshold level of the system. The threshold level is estimated based on the condition is maintained. The total observation in the channel is represented by the combination of $\mathrm{N} * \mathrm{p}$. but this observation is not reliable and negative effect is obtained in the system. The initialization of observation is done by using decoding process. The channel will ensure the probability of the system based on the receivers observation.

Coming to polar code encoder and decoder, they produce the logic which is very attractive in the system by high throughput. By using this system, less delay is obtained and noise is reduced. The standard polar code construction is used to get effective output. Here the channel is estimated based on the combination of various signals. The code construction process will map the system based on fading of signals. Here $\mathrm{N}$ copies are mixed together to get effective output.

The main intent of random interleaver is to rearrange the elements based on the vector format. The memory address is saved on the device using permuter. In the permuter, pseudo random gate permutation is performed. Based on the memory address, the data is arranged in the order of pseudo random.

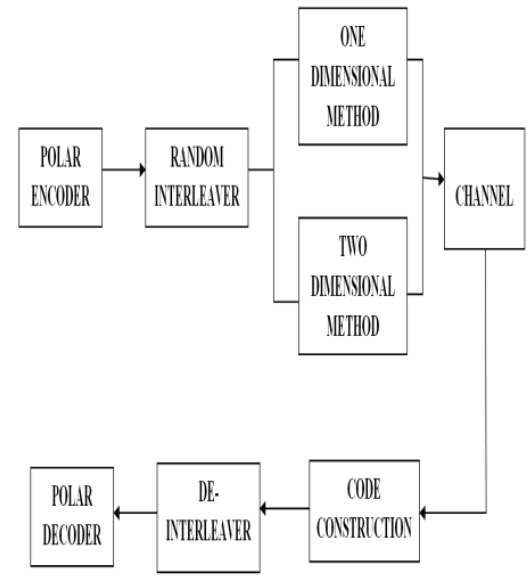

Fig. 3: SYSTEM MODEL
The bit error rate and noise is determined by using polarizing transformation. From below equation we can observe the approximation of sub channels in effective way. Here the same signal is received by using multiple channels.

$$
\widehat{s}=\sum_{j=0}^{\Lambda-1} h_{j} r_{j}=\underbrace{\left(\sum_{j=0}^{\Lambda-1} h_{j}^{2}\right)}_{\tilde{h}^{2}} s+\underbrace{\sum_{j=0}^{\Lambda-1} h_{j} \eta_{j}}_{\eta} .
$$

Bit error probability is maintained to express the system to determine the ratio. The sub channels are polarized after the transformation. The phase $2 \mathrm{i}$ is used to get the numerical calculations. Error probabilities are estimated to get the transformation using sub channels.

Number of indices are maintained to reduce the noise and error probability in the system. The polar code will use the channel which will map the system. Here two types of dimensional methods are used in the system, one is one dimensional method and another one is two dimensional methods. One dimensional method will determine the error probability in the system using fading channels. The interleaver will permute the data in effective way and in the same way de-interleaver will arrange the data to get back to the original state.

\section{NUMERICAL RESULTS}

To Evaluate the performance of proposed polar codes over AWGN \& Nakagami Channels we employed a symbol rate of 120k symbols for second and operated with code rate $\mathrm{R}=1 / 3$, list size $\mathrm{L}=8$ with crc length $=19$ at $\mathrm{Eb} / \mathrm{No}=4.5$ $\mathrm{db}$. The code length of polar codes achieves the capacity of an arbitory binary input memory less channel. Polar code is generated by ' $\mathrm{K}$ ' rows of generated matrix, where

$$
A=\left\{\begin{array}{ll}
1 & 0 \\
1 & 1
\end{array}\right\}
$$

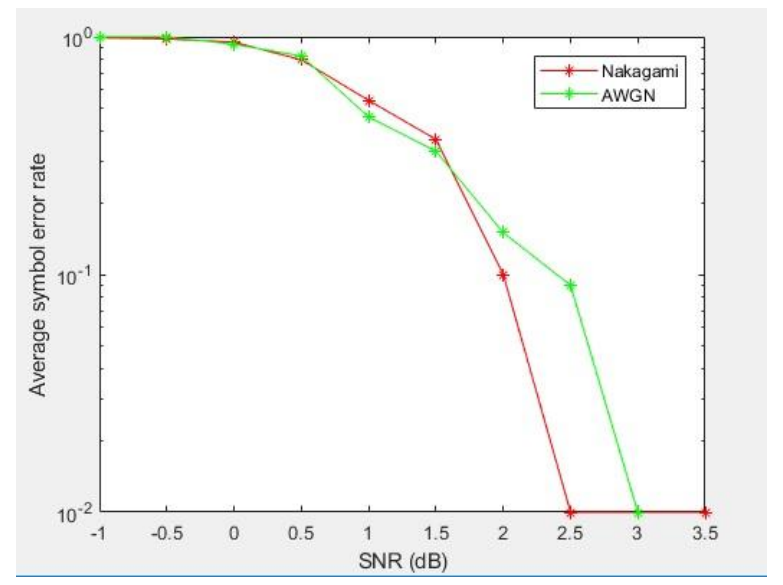

Fig. 4: Signal to Noise Ratio Vs Average Symbol Error Rate of proposed system

\section{CONCLUSION}

In this paper it is substantially we have shown the gain achieved in implementation of Nakagami Channel over 


\section{DESIGN OF POLAR CODES FOR RAYLEIGH FADING CHANNEL USING RANDOM INTERLEAVER AND DE-INTERLEAVER}

Rayleigh fading channel using random interleaver and deinterleaver. The random interleaver and de-interlaver will produce the signals for per mutational level. The channel acts as communication between polar encoder and polar decoder. Approximation of construction is introduced to construct the signals for better error performance. Hence polar code gives the performance in effective way depend on fading channel. Compared to other codes, the proposed polar code gives effective output.

\section{REFERENCES}

1. E. Arikan, "Channel polarization: A method for constructing capacity achieving codes for symmetric binary-input memoryless channels," IEEE Transactions on Information Theory, vol. 55, no. 7, pp. 3051-3073, July 2009.

2. P. Trifonov and V.Miloslavskaya, "Polar codes with dynamic frozen symbols and their decoding by directed search," in Proceedings of IEEE Information Theory Workshop, September 2013, pp. $1-5$.

3. V. Miloslavskaya and P. Trifonov, "Sequential decoding of polar codes," IEEE Communications Letters, vol. 18, no. 7, pp. 1127-1130, 2014.

4. I. Tal and A. Vardy, "How to construct polar codes," IEEE Transactions on Information Theory, vol. 59, no. 10, pp. 6562-6582, October 2013.

5. S.-Y. Chung, T. J. Richardson, and R. L. Urbanke, “Analysis of sumproduct decoding of low-density parity-check codes using a Gaussian approximation," IEEE Transactions on Information Theory, vol. 47, no. 2, February 2001.

6. P. Trifonov, "Efficient design and decoding of polar codes," IEEE Transactions on Communications, vol. 60, no. 11, pp. 3221 - 3227, November 2012.

7. Y. Zhang, A. Liu, K. Pan, C. Gong, and S. Yang, "A practical construction method for polar codes," IEEE Communications Letters, vol. 18, no. 11, November 2014.

8. J. Boutros and E. Biglieri, "Polarization of quasi-static fading channels," in Proceedings of IEEE International Symposium on Information Theory, 2013.

9. H. Si, O. O. Koyluoglu, and S. Vishwanath, "Polar coding for fading channels: Binary and exponential channel cases," IEEE Transactions On Communications, vol. 62, no. 8, August 2014.

10. A. Bravo-Santos, "Polar codes for the Rayleigh fading channel," IEEE Communications Letters, vol. 17, no. 12, December 2013.

11. I. Tal and R. Roth, "On list decoding of alternate codes in the Hamming and Lee metrics," in Proceedings of IEEE International Symposium on Information Theory, 2003.

12. K. Chen, K. Niu, and J. Lin, "Improved successive cancellation decoding of polar codes," IEEE Transactions on Communications, vol. 61, no. 8, pp. 3100-3107, August 2013. 